\title{
Évolution de la législation concernant les maladies professionnelles considérées comme provoquées par les rayonnements ionisants
}

\author{
J. HÉBERT ${ }^{1}$
}

(Manuscrit reçu le 15 janvier 2003, accepté le 4 décembre 2003)

\begin{abstract}
RÉSUMÉ Après rappel de l'histoire et de l'économie du système classique français d'indemnisation des maladies professionnelles, les conditions d'insertion de celles de ces maladies qui peuvent être provoquées par les rayonmements ionisants sont examinées. L'évolution de la législation et de la jurisprudence les concernant est étudiée, pour conclure par l'examen des solutions que le nouveau système institué par la loi du 27 janvier 1993 pourrait apporter dans certains cas.
\end{abstract}

ABSTRACT Evolution of the legislation concerning the professional diseases considered as produced by ionised radiation.

After having remind the history and structure of the classical French system of compensation of occupational diseases, conditions put to insert such of these diseases induced by ionizing radiations are studied, before to conclude by an exam of solutions that a new system introduced by an act of 27 of January 1993 could offer in some situations.

\section{Historique et économie de la législation des maladies professionnelles}

Rappelons que la loi du 9 avril 1898 avait instauré en France une réparation des accidents du travail. Cette réparation, fondée sur une présomption d'imputabilité du préjudice corporel à l'occupation professionnelle, était forfaitaire et excluait, en principe, une réparation principale ou complémentaire fondée sur la responsabilité civile de l'employeur. Il a fallu attendre plus de $20 \mathrm{ans}^{2}$ pour que la loi du 25 octobre 1919 institue, et seulement pour deux maladies (saturnisme et hydrargyrisme) exclusivement causées par l'exposition professionnelle, un régime comparable

25 chemin du Trou Marlin, 78380 Bougival, France.

A rapprocher des lois du 23 avril 1919 instituant la journée de 8 heures et du 25 mars 1919 sur les conventions collectives. Sur la question des maladies professionnelles, il convient de noter qu avant 1919 les mineurs, par la loi du 29 juin 1894 . les inscrits maritimes, par une loi du 21 avril 1898 et les pompiers, par un décret du 12 juillet 1899, bénéficiaient déjà de régimes d’indemnisation. Le régime minier a conservé après 1946 une certaine autonomie, qui se traduit notamment dalns les statistiques. 
d'indemnisation. Est-il besoin de rappeler les espoirs suscités par la révolution d'octobre, galvanisant la vieille tendance révolutionnaire du mouvement ouvrier, qui s'étaient traduits en France par de grandes grèves? Le gouvernement Clemenceau donna du «grain à moudre » à l'autre tendance réformiste, notamment en mettant fin, en apparence tout au moins, aux atermoiements sur l'adaptation aux maladies professionnelles, du système institué en 1898 (Dolléans, 1953 ; Bonnefous, 1958 ; Braudel et Labrousse, 1979). En effet, la France n'était pas en avance en ce domaine, ayant été précédée par la Suisse (1877) et la GrandeBretagne (1897 et 1900) et son antériorité sur l'Allemagne (1925) n'était qu'apparente, avec deux tableaux seulement contre une quinzaine, dont les maladies occasionnées par les « rayons $X$ et toute autre énergie radioactive».

Le bénéfice de la loi de 1919 découle d'une présomption d'imputabilité de la maladie au travail habituel effectué par le salarié. La loi impose en effet de tenir pour causées par le travail certaines maladies, si certaines conditions sont remplies, conditions qui ont été reconnues par la loi comme statistiquement liées à une fréquence particulière de ces maladies dans certaines industries, alors qu'en droit civil le demandeur aurait dî prouver que son préjudice avait été causé par l'inobservation à son égard et dans son cas particulier d'une obligation, par exemple de sécurité, par son employeur, co-contractant du contrat de travail ou par une « chose », rayonnement par exemple, « sous la garde » d'un tiers.

Mais en contrepartie, comme pour les accidents du travail, la réparation prévue par la loi de 1919 était forfaitaire, et à la charge de l'employeur. Le travailleur, qui voit sa maladie reconnue comme d'origine professionnelle, ne peut obtenir de réparation plus complète, qu'en cas de faute inexcusable ou, sur la base du droit civil, que dans l'hypothèse heureusement rare de faute intentionnelle de l'employeur.

Le critère de distinction entre la «maladie professionnelle » et l' « accident du travail » est, selon l'article L 461-2 actuel du code de la sécurité sociale, que les « maladies professionnelles» sont les « manifestations morbides d'intoxications aiguës ou chroniques présentées par les travailleurs exposés d'une façon habituelle à l'action des agents nocifs mentionnés aux tableaux qui les énumèrent » et qui, dans le cas notamment du tableau $n^{\circ} 6$, donnent, à titre indicatif, la liste des principaux travaux comportant la manipulation ou l'emploi de ces agents nocifs. $\mathrm{Au}$ contraire, une affection pathologique n'ouvre droit à réparation à titre d'accident du travail que si elle a sa source dans une lésion apparue soudainement au temps et au lieu du travail et à laquelle on peut donc assigner une origine et une date certaine. Si bien que par exemple le tétanos, la syphilis ou le sida contractés à la suite d'une blessure survenue à l'occasion du travail en un jour et lieu déterminés sont des accidents du travail et non des maladies professionnelles. 
Le lableau précise également un délai de prise en charge courant entre la cessation d'exposition au risque et la constatation médicale de l'affection considérée. Ce délai de prise en charge doit être distingué d'un délai de prescription également prévu.

Par la loi $\mathrm{n}^{\circ}$ 46-2426 du 30 octobre 1946, ce système a été intégré dans le régime de sécurité sociale, les organismes de sécurité sociale étant substitués à l'employeur dans la charge de la réparation. La loi n 93-121 du 27 janvier 1993 a permis de présumer aussi l'origine professionnelle des maladies « directement causées par le travail habituel de la victime " mais ne satisfaisant pas à d'autres conditions légales ou des maladies caractérisés non désignées dans un tableau mais « essentiellement et directement causées par le travail habituel » sur avis d'un comité régional de reconnaissance des maladies professionnelles.

\section{Insertion des maladies provoquées par les rayonnements ionisants dans le régime des maladies professionnelles}

La loi du $1^{\text {cr }}$ janvier 1931 (JO 4 janvier 1931) a facilité la reconnaissance des maladies professionnelles, en desserrant quelques freins posés par la loi de 1919 et surtout a ajouté quatre nouveaux tableaux, en particulier un tableau $n^{\circ} 6$ sur les affections provoquées par les rayons $X$ ou le radium ${ }^{3}$. Le nombre de salariés exposés à ces rayonnements était sans doute alors très faible, et des maladies comme la silicose. qui faisaient de nombreuses victimes, ne bénéficiaient pas encore d'un tableau (il a fallu attendre jusqu'en 1945). Aujourd'hui il existe 98 tableaux.

C'est en effet au cours des années 20, que l'on pris conscience des risques présentés par les rayonnements ionisants pour les travailleurs (en 1924 par la mise en évidence de cancers des os de la mâchoire chez les jeunes femmes employées à la peinture des cadrans dans l'industrie de guerre américaine, les décès d'anémie ou leucémie d'assistants de Marie Curie en 1922-24, et d'autres publications à l'étranger). Un décret du 19 février $1927^{4}$. articles 1 et $\mathrm{K}$, imposait déjà aux médecins de déclarer, parmi les maladies ayant un caractère professionnel, notamment celles causées par les «l'action des rayons $\mathrm{X}$ et des substances radioactives » (Reid, 1974). Mais le projet de loi déposé par le gouvernement le

Les uableaux $n^{\circ} 3,4$ el 5 concerment le tétrachloréthanc. le hencène el le pluosphose.

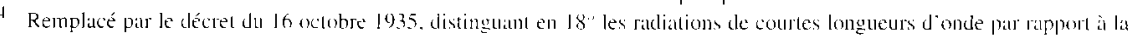

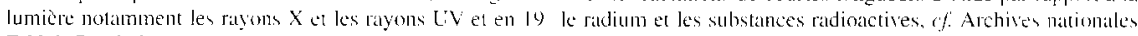
$\mathrm{F} 22217 \mathrm{cl} 218$. 
27 février 1927 ne prévoyait pas de tableau pour les rayons X. C'est la commission d'assurance et de prévoyance sociales de la Chambre des députés qui, dans ses rapports préparatoires de la loi de 1931 s'est prononcé en faveur de l'extension aux rayons $\mathrm{X}^{5}$. Après avoir rappelé le « glorieux martyrologe » des radiologues, elle a insisté sur la nécessité de protéger aussi les chimistes et divers ouvriers fabriquant les tubes de Crookes ou les peintres «qui se servent de produits lumineux radioactifs comme cela se pratique en Amérique (nécrose des maxillaires par phosphorescence du sulfure de zinc) ${ }^{6}$. La commission des maladies professionnelles, crée par la loi de 1919, n'a pas accepté d'autres adjonctions aux deux tableaux de la loi de 1919 que celles proposées par le projet de loi (les actuels tableaux $n^{\circ} 3,4$ et 5) et celle concernant les rayons $X$, repoussant d'autres projets de tableaux proposés par la commission parlementaire. On peut supposer que le patronat, influent dans cette commission, ait pensé devoir jeter du lest sur les affections causées par les rayons X, qui étaient sous les projecteurs de l'actualité, afin de sauvegarder par ailleurs les intérêts de l'industrie chimique en gagnant quelques années pour l'instauration (en décembre 1938 au plus tôt) de tableaux concernant les maladies provoquées par divers produits chimiques.

Dans cette première version du tableau nº 6, annexé à la loi du $1^{\mathrm{er}}$ janvier 1931 , on trouve radiodermites, cancer des radiologistes, anémie simple et pernicieuse, leucémie et radionécrose osseuse. On peut s'étonner de l'absence d'affections oculaires, bien que Marie Curie ait été déjà pratiquement aveugle à cette époque. Les délais dits de « responsabilité » sont courts, comparés aux délais actuels : un an, y compris pour la radionécrose osseuse, sauf pour les cancers des radiologistes pour lesquels le délai est de 5 ans. La liste des « travaux susceptibles de provoquer ces maladies » parait englober les travaux qui, à l'époque, exposaient aux rayons $\mathrm{X}$ et substances radioactives et n'est pas limitative, car elle se termine par la formule «travaux dans toutes les industries ou commerces utilisant les rayons $\mathrm{X}$ et les substances radioactives $»^{7}$.

\footnotetext{
5 Un sondage dans les travaux préparatoires a été effectué : rapport de la commission d'assurance et prévoyance sociale de la Chambre des députés du 31 mai 1929 annexe 1723, JO 13 novembre 1929, p. 74. Ce rapport cite à l'appui de sa proposition d'étendre le chanp d'application de la loi aux rayons X un rapport du Dr Simone Laborde à la commission supérieure des maladies professionnelles, et le rapport de la Commission d'hygiène. assistance, assurance el de la prévoyance sociale du Sénat $n^{\circ} 352$ de 1926. Les dossiers sur l'application de la loi du 25 ostobre 1919 jusqu’à 1936 déposés aux Archives nationales sous les cotes F 22517 a 519 ne contiennent malheureusement pas l’avis de la Commission supérieure et ni le rapport médical précités.

6 Le rapport $n^{\circ} 1723$ cite le cas des chimistes Demelander et Demenitroux, anciens assistants de Marie Curie (il y aurait eu une décision judiciaire, nori-publiée) et celui des peintres américains «victimes expiatoires du progrès». Il ajoute curieusement à la liste des travailleurs exposés «les ouvriers soudeurs aux rayons ultra-violets. lęs cinéastes et tous les Inanipulateurs d'infra-rouges ou d'ultra-violets ». Cet intérêt précoce pour les rayonnements non-ionisants est à noter, car ils ne semblent pas faire l'objet de tableaux aujourd'hui encore.

7 Le droil actuellement en vigueur distingue les tableaux qui, comme le tableau $\mathbf{n}^{\circ} 6$, concernent des affections présumées provoquée par des agents nocits et pour lesquelles la liste des travanx susceptibles de provoquer ces maladies n'est qu'indicative et les tableaux concemant des infections microbiennes ou présumées résultant d’un ambiance ou d’attitude particulières dans lesquels les trávaux sont limitativement énumérés.
} 


\section{3. Évolution de la législation concernant les maladies professionnelles provoquées par les rayonnements ionisants}

Les révisions du tableau $n^{\circ} 6$ intervenues entre ce texte de 1931 et celui en vigueur de nos jours ( $\mathrm{D} \mathrm{n}^{\circ} 84-492$ du 22 juin 1984) sont les suivantes :

(a) Décret $n^{\circ} 46-1959$ du 31 décembre 1946 (JO $1^{\text {er }}$ janvier 1947). Le tableau $n^{\circ} 6$ reprend exactement celui du $1^{\text {er }}$ janvier 1931.

(b) Décret $n^{\circ} 50-1533$ du 9 décembre 1950 (JO 13 décembre 1950, p. 12624). Dans la colonne des affections, on note que :

- les « radiodermites et radiumdermites » sont remplacées par « lésions aiguës de la peau et des muqueuses » avec un délai de prise en charge de 10 ans au lieu d'un an,

- le «cancer des radiologistes » devient le «cancer broncho-pulmonaire avec inhalation » avec un délai de 20 ans au lieu de 5 ans,

- « l'anémie simple avec leucopénie » devient « l'anémie progressive légère de type hypoplasique ou aplasique », avec un délai d'un an, et elle est distinguée de la « leucopénie avec neutropénie », également affectée d'un délai d'un an, - «l'anémie pernicieuse » devient «l'anémie progressive grave de type hypoplasique ou aplasique », avec un délai de 3 ans,

- la « radionécrose osseuse », dont le délai passe de 1 à 5 ans, se distingue désormais du « sarcome osseux », dont le délai est de 15 ans, le plus long du tableau,

- mais le tableau de 1950 ajoute en outre le « syndrome hémorragique », avec le délai d'un an, ainsi que, pour la première fois, des affections oculaires : blépharite ou conjonctivite, délai de 7 jours, kératite, délai d'un an et cataracte, avec un délai de 5 ans.

En ce qui concerne la colonne des «travaux susceptibles de provoquer ces affections ", on assiste à l'introduction, comme pour d'autres tableaux, d'un premier alinéa « tous travaux exposant à l'action des rayons $\mathrm{X}$ ou des substances radioactives naturelles ou artificielles ou à toute autre source d'émission corpusculaire ». Mais, dans notre cas, cette formulation, prise à la lettre, pourrait s'appliquer à n'importe qu'elle activité puisque la vie sur la terre comporte une exposition aux rayonnements du soleil ou de la terre ! Elle fait quasi double emploi avec les deux derniers alinéas, repris en substance du décret de 1946 et de la loi de 1931, de « travaux [...] dans les hôpitaux, les cliniques, les cabinets médicaux, les cabinets dentaires et radiologiques, dans les maisons de santé et les centres anticancéreux » (les hôpitaux ont été ajoutés par ce décret de 1950) et les «travaux dans toutes les industries ou tous commerces utilisant les rayons $\mathrm{X}$ et les substances radioactives » en y ajoutant « les substances ou dispositifs émettant les rayonnements indiqués ci-dessus ». Entre l'alinéa $1^{\text {er }}$ et ces deux derniers est 
insérée une liste plus détaillée de travaux, reprenant à quelques détails près celles de 193 I et 1946 , et qui est non limitative, car commençant par «notamment», avec cependant une suppression, celle de la « vente et location de radium et des substances radioactives $\%$. On notera que la seule industrie nucléaire citée est l'«extraction et traitement des minerais radioactifs » déjà mentionnée en 1931 et 1946. Avec la « préparation et application de produits luminescents radifères »ce sont les seules activités étrangères à la médecine ou la recherche, qui demeurent dominantes. Mais cela est sans conséquences juridiques, puisqu'il s'agit d'une liste indicative.

(c) En effet, par le décret n ${ }^{\circ}$ 55-1212 du 13 septembre 1955 (JO 15 septembre 1955, p. 9677), l'en-tête de la $3^{\mathrm{e}}$ colonne du tableau est, comme dans les autres tableaux concernant les intoxications provoquées par des agents nocifs mentionnés par ces tableaux, modifié et devient «liste indicative des principaux travaux susceptibles de provoquer ces maladies ».

(d) Décret $\mathrm{n}^{\circ}$ 60-1081 du $1^{\mathrm{er}}$ octobre 1960 (JO 11 octobre 1960, p. 9280) remplace et développe les rubriques « leucoses ou états leucémoïdes » et « lésions aiguës de la peau et des muqueuses » par les six suivantes : leucoses (avec un délai de 10 ans); états leucémoüdes (délai : 3 ans); radiodermite aiguës (délai : 60 jours) ; radiodermite chroniques (délai : 10 ans) ; radio-épithélite aiguë des muqueuses (délai : 60 jours) ; radio-lésions chroniques des muqueuses (délai : 5 ans).

(e) Décret $\mathrm{n}^{\circ}$ 63-405 du 10 avril 1963 (JO 20 avril 1963) ajoute les travaux exposant aux rayonnements dans les sanatoriums, manifestant de nouveau le souci de n'omettre aucun lieu d'exercice de l'irradiation à fin médicale.

(f) Décret $n^{\circ} 84-492$ du 22 juin 1984 (JO 26 juin 1984, p. 1972) qui est le texte aujourd'hui en vigueur. Le Dr $\mathbf{H}$. Jammet et le professeur Jean Chanteur (SCPRI) ont beaucoup cuvré pour cette mise à jour notamment en ce qui concerne l'abandon de la rubrique concernant les «états leucémoïdes », insuffisamment précise.

Les affections sanguines font de nouveau l'objet d'une rédaction nouvelle : « anémie, leucopénie, thrombopénie ou syndrome hémorragique », dont le délai, s'ils sont consécutifs à une irradiation aiguë, est de 30 jours et d'un an s'ils sont consécutifs à une irradiation chronique. D'autres délais sont allongés : de 5 à 10 ans pour la cataracte, de 10 à 50 ans pour le cancer broncho-pulmonaire par inhalation, avec la qualification nouvelle de «primitif», de 10 à 50 ans pour le sarcome osseux. Ce délai de 50 ans pourrait être le plus long prévu par cette législation des maladies professionnelles.

Bien entendu, ces listes de maladies et leur évolution mériteraient un examen par un médecin au fait de l'évolution des connaissances en ce domaine. 
L'énumération des travaux n'a pas changé substantiellement depuis 1931 et ignore toujours l'industrie du cycle du combustible nucléaire et la production d'électricité par réacteur. Les réacteurs de recherche et les accélérateurs de particules, à des fins scientifiques ou industrielles, ne sont pas non plus mentionnés expressément.

\section{La jurisprudence concernant les maladies du tableau $n^{\circ} 6$}

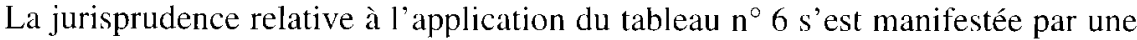
séparation entre la réglementation de la radioprotection et celle de la réparation des maladies professionnelles, exprimée en particulier par l'arrêt dame Majoni (Cass. Soc. 10 février 1966 et Paris 4 décembre 1965$)^{8}$. Ce qui ouvre la possibilité de voir reconnue comme d'origine professionnelle une maladie figurant sur la liste, bien que le respect de la réglementation de la radioprotection et en particulier le respect des limites de doses relevées par la dosimétrie obligatoire, n'aient pas été contesté.

Dans son arrêt Majoni, la Cour de cassation constate qu'«aucun degré d'intensité ou de dose dangereuse n'ayant été prévu » (par le tableau 6), et exige pour combattre la présomption d'origine la «preuve d'une cause autre que la radioactivité à laquelle (la dame Majoni) était exposée du fait de son emploi et que sans aucun doute possible ce risque n' a eu aucune influence sur son origine ou son évolution ", bref c'est une exigence de probatio quasi diabolica en présence

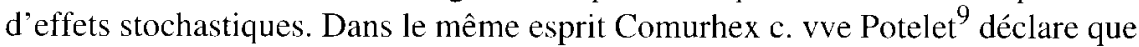
la victime a été « exposée plusieurs années, dans le cadre de son travail, à l'action des rayonnements ionisants [...] peu important le taux d'irradiation ». Par contre la Cour de cassation exige le respect strict de la définition de la maladie donnée par le tableau. Ainsi elle ne reconnaît pas comme maladie du tableau $n^{\circ} 6$ un cancer broncho-pulmonaire ne résultant pas d'une inhalation de substances radioactives, mais survenue après diffusion dans l'organisme de métastase d'un cancer des testicules $^{10}$.

La discussion s'est cantonnée le plus souvent en l'examen des conditions mêmes du tableau $n^{\circ} 6$ sur le point de savoir si le travail exécuté entraînait ou non une « exposition habituelle », c'est-à-dire, à examiner si le cas entrait ou non dans le cadre de la définition légale de la maladie professionnelle. Déjà dans l'affaire

\footnotetext{
8 Commenté pal Toutée et de Nercy. À propos d'un cas de maladie professionnelle auribuée aux rayonnements ionisants, droit sccial $1967 n^{\circ}$ l p. 51 et seq. ; J. Hébert, note sous Cass. Soc. 10 février 1966 J.C.P. 1967 II 14923. L'avocat général avail escimé que l'accueil de « la notion de seuil de nocivité sonnerait le glas du régime actuel des maladies professionnetles » en conduisant les tribunaux à arhitrer des conilits purement scientifiques entre experts D 1966.305. Par contre. la clane Majoni a perclu son action au civil sur le terain de la faute inexcusable. Paris 8 février 1969 et 12 mars 1969. J.C.P. 1969 I 16089 note J. Vergne.

y Cass. Soc. 6 mai 1993 arrêt n 1619

in Cass. 21 octobre 1985 dame Giret-Gaz. Pal. tableaux de jurisp. 1986, p. 9.
} 
dame Majoni, la Cour de cassation a admis « l'exposition de façon habituelle, du fait de son travail, à l'action des substances radioactives naturelles ou artificielles ». De même dans l'affaire d'une documentaliste de l'IN2P3 dont le poste de travail ne se trouvait pas isolé de sources "' . Dans l'affaire George, la Cour le 24 novembre $1976^{12}$ a estimé également que, du fait du passage devant une source pour se rendre à son poste de travail, il avait été « effectivement et de façon habituelle exposé aux rayonnements ionisants ». Par contre dans l'affaire Saltel la Cour de cassation Soc. le 18 juin $1970^{13}$ a approuvé la Cour d'appel d'avoir estimé que des «expositions isolées et non notables» ne constituaient pas une « exposition habituelle ». De même le 25 février 1982, la Cour d'appel de Paris (Hébert, 1983) a estimé qu'il va de soi que l'exposition pour être habituelle « doit nécessairement présenter des conditions minimales de fréquence, de durée et d'intensité ».

Mais au moins jusqu'à l'adoption du système mixte toute demande d'indemnisation ne satisfaisant pas à toutes les conditions citées supra de la présomption d'origine était rejetée, (cf. infra le cas de dame Kornacker).

Le travailleur victime d'une maladie professionnelle peut obtenir une majoration de rente et la réparation des préjudices non couverts par les prestations sociales (pretium doloris, esthétique, perte de possibilité de promotion professionnelle) en cas de faute inexcusable de l'employeur (acte ou omission volontaire d'une gravité exceptionnelle, conscience du danger que devait avoir son auteur, absence de toute cause justificative). La dame Majoni n'a toutefois pas gagné sur ce terrain (Paris 12 mars 1969) ${ }^{14}$. La victime peut également se porter partie civile en cas de poursuites pénales contre l'employeur, pour faire reconnaître une faute intentionnelle. Ainsi dans la pénible affaire de Forbach, mais la Cour de Metz, 17 février 1994, n'a accordé que le remboursement de leur frais de procédure aux salariés victimes de cet accident.

\section{Observations finales}

Reste que le système de liste français, s'il présente un avantage de simplicité pour les cas satisfaisant les conditions de la présomption, englobe implicitement des cas pour lesquels la causalité professionnelle est douteuse. Ce qui est le cas de l'application à un individu déterminé de résultats, de nature statistique, concernant des effets stochastiques plus marqués dans un isolât de population que dans la

\footnotetext{
1 Cass. Soc. 2 mars 1978, semaine juridique 1978-2, p. 139.

12 Cass. Soc. 24 novembre 1976 Bull. Cass. 1976 n 622-semaine juridique 1977-IV-9.

13 Cass. Soc. Bull. 197। V 430 et D.S. 1971-I-290

14 JCP 1969-I-16089 note J. Vergne.
} 
population générale. Tant que les statistiques ${ }^{15}$, ne sont pas utilisées indûment, cela paraît supportable. Il est clair d'ailleurs que les tableaux ne peuvent être révisés qu'à des intervalles plus ou moins éloignés et il est possible qu'entre temps des soupçons se portent, puis se confirment, sur le lien de nouvelles affections avec tel ou tel agent nocif, ou sur les délais de leur apparition après la cessation d'exposition au risque. On en a un bon exemple avec le sarcome osseux dont le délai de prise en charge a été porté de 15 à 50 ans entre le tableau de 1950 et celui du 22 juin 1984. Il existait donc un « angle mort " ${ }^{16}$, ou des situations entre deux chaises, le régime de l'accident du travail d'une part et celui des maladies professionnelles d'autre part. On a discuté du point de savoir si le salarié pouvait dans ce cas obtenir réparation, sur la base du droit civil, de la responsabilité délictuelle ou quasi délictuelle (articles 1382, 1383 et 1384) ou contractuelle. Dans l'affaire dame Kornacker contre Cie générale de radiologie ${ }^{17}$ il en a été ainsi jugé parce que la maladie de la victime n'avait pas encore été classée professionnelle à l'époque des faits. Étant entendu que la Cour de cassation s'est prononcée nettement en faveur du «non cumul », et n'admet pas, sauf faute intentionnelle, une action au civil parallèle à la demande de réparation prévue par le code de la sécurité sociale, ou que le salarié ait le choix entre les deux voies, celle du droit civil ou celle du droit social ${ }^{18}$.

Aussi, une tendance s'est dégagée dans la Communauté européenne d'abord en faveur de la généralisation du système dit mixte, d'origine allemande, qui à côté de présomptions d'origine trouvant leur fondement dans des listes d'affections, de travaux exposant et de délais, comme dans le système exposé ci-dessus, ouvre en outre au salarié la possibilité de voir reconnattre comme d'origine professionnelle une affection ne satisfaisant pas l'un ou l'autre de ces critères, a condition bien entendu de prouver l'existence de ce lien causal, puis en faveur d'une appréciation par des médecins.

La Commission a procédé par voie de recommandations (qui ne lient pas les États membres). Ainsi la recommandation du 23 juillet 1962 (JOCE, 1962), concernant l'adoption d'une liste européenne des maladies professionnelles, préconise l'introduction dans les législations nationales de dispositions permettant

\footnotetext{
15 Caisse nationale de l'ussurance maladie des travailleurs salariés, statistiques financières et technologiques des accidents du travail. publiées annuellement.

16 Dupeyroux, un critère de l'accident du travail par rapport à la maladie D. $1966 \mathrm{~J} 251$ et notion d'accident du trawail D 1964 chi. V.

17 Cass. Soc. $1^{\text {er }}$ mars 1956. J.C.P. 1956 II 9619 et Orléans 18 février 1959, J.C.P. 1959 II 11053 . La Cour de cassation avait jugé repoussé l'action én croyant à tort à une prescription au titre de la loi de 1919. En fait la maladie dont a soulfert dame Kornacker n’élait pás encore reconnue comme professionnelle à l'époque des faits et la cour de renvoi a jugé qu’à « alucune disposition |...| ne prive la victime d'un dommage (ne pouvant se prévaloir de la législation forlaitaire) d’invoquer à l'égard de son employeur les dispositions de l'arcicle 1382 et de l'article $1384 \$ 1^{\text {Lr }}$ du Code civil [...] le préposé qui manjpule la chose qui a causé le dommage n’en devenant pas pour autant gardien "

18 Cass. 13 juillet $1993 n^{\circ} 45960$ et 11 octobre 1994 RJS $11 / 94 n^{\circ} 1307$.
} 
l'indemnisation de travailleurs atteints de maladies non-inscrites sur les listes nationales, mais dont l'origine professionnelle est prouvée et à supprimer, sauf exceptions, le caractère limitatif des conditions (symptomatologie, énumération des activités, durée minimale d'exposition au risque, délai maximal de prise en charge) pour donner plein effet à une appréciation par les médecins compétents. La recommandation 66/462 du 20 juillet 1966 (JOCE 147 du 9/08/66 p. 2696) reprenait et complétait ces lignes directrices en prévoyant des notices destinées aux médecins et experts sur les maladies figurant sur la liste européenne. La recommandation 90/326/CEE du 22 mai 1990 (JOCE n ${ }^{\circ} \mathrm{L}$ 160/39 du 26/06/90) adopte une liste européenne des maladies professionnelles, dans laquelle, sous le $\mathrm{n}^{\circ} 508$, figure les «maladies provoquées par les radiations ionisantes » et en annexe un aperçu de la situation dans les États membres.

En France la jurisprudence a tenu compte de cette évolution. Ainsi la Cour d'appel de Limoges a pu juger le 5 juin $1992^{19}$ que « les juridictions sociales ont le pouvoir de déterminer, après expertises médicales, si une affection particulière entre dans la définition de l'une des maladies professionnelles. Tel est le cas de la maladie de Vasquez, qui entre dans le cadre du tableau n ${ }^{\circ} 6$ sous le vocable général de leucémie ».

Surtout, la loi $n^{\circ} 93-121$ du 27 janvier 1993 article 7-1 a modifié l'article L 461-1 du Code de la sécurité sociale en disposant d'une part que «si une ou plusieurs conditions tenant au délai de prise en charge, à la durée d'exposition ou à la liste limitative des travaux ne sont pas remplies, la maladie telle qu'elle est désignée dans un tableau de maladies professionnelles peut être reconnue d'origine professionnelle lorsqu'il est établi qu'elle est directement causée par le travail habituel de la victime »; d'autre part que «peut être également reconnue d'origine professionnelle une maladie caractérisée non désignée dans un tableau [...] lorsqu'il est établi qu'elle est essentiellement et directement causée par le travail habituel de la victime et qu'elle entraîne le décès de celle-ci ou une incapacité permanente d'un taux évalué dans les conditions mentionnées à l'article L 434-2 (66,6\%) et au moins égal à un pourcentage déterminé ». L'harmonisation européenne est cependant obérée par la plus ou moins grande exigence des organes d'examen et tribunaux en matière de preuve de la causalité, particulièrement délicate s'il s'agit d'un effet stochastique. En France, il semble que le nombre de cas fondés sur les deux alinéas précités soit inférieur à ce qui était prévu.

L'article L 461-8 du code de la sécurité sociale a prévu d'accorder une indemnité spéciale au travailleur atteint de certaines affections consécutives à l'inhalation de silice libre, de poussières d'amiante ou pour prévenir l'aggravation

19 Somm. SS. avril 1993 p. 48603. 
de son état en cas de sidérose professionnelle, tandis que la Cour de cassation, par un arrêt du 28 février 2002 élargissait la notion de faute professionnelle à propos de maladies provoquées par l'amiante. Divers rapports ont montré que le caractère forfaitaire de l'indemnisation des maladies professionnelles aboutissait à une réparation inférieure à celle attribuée en cas d'accidents de la route. L'avenir dira si, malgré les difficultés actuelles, une meilleure indemnisation sera accordée aux maladies du tableau $n^{\circ} 6$.

Signalons également qu'en vertu de l'article L 461-6 (ex L 500) du code de la sécurité sociale, tout docteur en médecine doit déclarer «toute maladie dont il a connaissance qui présente à son avis un caractère professionnel $»$. Le décret $n^{\circ} 63$ 865 du 3 août 1963 (JO 23/8/63) précise en annexe « maladies susceptibles d'avoir un caractère professionnel et d'être imputées [...] aux rayonnements ionisants ».

En vertu du Code rural, les salariés du régime agricole bénéficient aussi d'une couverture du risque de maladies professionnelles. Un tableau $n^{\circ} 20$ énumère les affections provoquées par les rayonnements ionisants, les délais de prise en charge et la liste indicative des travaux, comme dans le système classique des salariés de l'industrie. Toutefois la liste des travaux est plus ciblée : «travaux effectués dans les services médicaux ou médico-sociaux ou dans les laboratoires » et « travaux concernant la conservation et l'analyse de produits agricoles divers ».

Pour mémoire, indiquons aussi que les travailleurs de l'État bénéficient des dispositions du code des pensions civiles et militaires (L 64-1339 du 26/1 1/1964 et $\mathrm{D} \mathrm{n}^{\circ}$ 68-756 du 13/08/1968) au lieu du régime des maladies professionnelles cidessus exposé. La loi de finances du 26 décembre 1959 introduit également un article 23 bis dans le statut général des fonctionnaires de l'Ordonnance du 4 février 1959, pour instituer une allocation temporaire d'invalidité en cas d'accident ou de maladie de service.

\section{RÉFÉRENCES}

Bonnefous E. (1958) Histoire politique de la $3^{\varkappa}$ République, tome ПI, p. 30 . PUF, Paris.

Braudel F., Labrousse E. (1979) Histoire économique et sociale de la France, tome IV, pp. $925,931$. PUF, Paris.

Dolléans E. (1953) Histoire du mouvement ouvrier, tome II, p. 299. A. Colin Ed. (seq. sur les grèves du printemps 1919).

Hébert J. (1983) Observations sur un arrêt rendu en matière de maladies professionnelles, Radioprotection $18(1)$, cet arrêt est inédit à ma connaissance.

JOCE (1962) n 080 du 31 août 1962 p. 2188 qui vise les maladies provoquées par les radiations ionisantes en Fl de la liste européenne.

Reid R. (1974) Marie Curie derrière la légende, p. 274. Seuil, Paris (seq. Albert Laborde collaborateur de Pierre et Marie Curie qui annonça à cette dernière en 1925 la mort d'anémie d'un de ses assistants était l'époux du Dr Simone Laborde citée en note 5. Cette dernière était une collaboratrice de Gustave Roussy.). 